\title{
Premières observations sur la santé des réfugiés syriens au Canada
}

\author{
Hansen $L^{1}$, Maidment $L^{1 *}$, Ahmad $R^{1}$
}

\section{Résumé}

Entre le 4 novembre 2015 et le 29 février 2016, un total de 26166 réfugiés sont arrivés au Canada. De ce nombre, seulement deux $(0,01 \%)$ réfugiés ont présenté des signes d'une maladie à déclaration obligatoire lors de l'examen médical aux fins de l'immigration (EMI), et ces personnes ont été aiguillées vers des services de santé publique aux fins de suivi. La majorité des réfugiés - 24640 (94.17\%) - étaient sur des vols nolisés coordonnés par le gouvernement et ont été soumis à un dépistage soutenu. Deux cent soixante-quatorze $(1,11 \%)$ réfugiés ont été évalués par des agents de quarantaine à leur arrivée au Canada, pour vérifier des signes d'une possible maladie transmissible (comme la fièvre), et dix $(0,04 \%)$ ont été envoyés à l'hôpital. Le personnel paramédical sur place dans les aéroports a aussi évalué 1212 (4,92 \%) réfugiés, dont 54 (0,22 \%) ont été transférés à l'hôpital; bon nombre d'entre eux nécessitaient des soins médicaux d'urgence.

Les autorités de santé publique provinciales et locales et les réseaux communautaires ont joué un rôle déterminant dans la prestation des soins de santé immédiats et à plus long terme aux réfugiés arrivant au Canada. Les deux besoins les plus pressants en matière de soins étaient la mise à jour de la vaccination et les soins dentaires. Comme certains réfugiés sont arrivés au Canada au plus fort de la saison grippale, un certain nombre ont présenté des infections des voies respiratoires supérieures d'une durée limitée. Lorsque le Haut Commissariat des Nations Unies pour les réfugiés (UNHCR) a communiqué avec les autorités canadiennes, il les a informées que les réfugiés syriens pourraient avoir de grands besoins en soins médicaux. Ces besoins n'avaient pas nécessairement été déterminés au préalable et ils pouvaient être liés à des problèmes tels que le diabète, des déficiences sur le plan du développement, des traumatismes liés au conflit ou des problèmes de santé mentale. Ces besoins en soins de santé des Syriens seront établis et pris en compte à mesure que ces personnes seront intégrées à leur système local de soins de santé.

L'arrivée des réfugiés syriens au Canada n'a pas suscité de préoccupations pressantes ni nécessité d'interventions urgentes sur le plan de la santé publique. L'expérience du Canada à ce jour indique que l'arrivée des réfugiés syriens au pays peut être prise en charge d'une manière qui permet d'intégrer ces réfugiés dans le système de soins de santé, sans que cela ne présente de risque accru pour la santé publique.

\section{Affiliation}

'Direction générale de l'infrastructure de sécurité sanitaire, Agence de la santé publique du Canada, Ottawa (Ontario)

*Correspondance : leanne. maidment@phac-aspc.gc.ca

Citation proposée : Hansen L, Maidment L, Ahmad R. Premières observations sur la santé des réfugiés syriens au Canada. Relevé des maladies transmissibles au Canada 2016;42-Suppl 2:S9-12.

https://doi.org/10.14745/ccdr.v42is2a03f

\section{Introduction}

Les considérations liées à la santé occupaient une place importante dans le plan du Canada d'accueillir 25000 réfugiés syriens entre décembre 2015 et le début de 2016. Ce plan avait été conçu de manière à accueillir les réfugiés et à les intégrer aux systèmes locaux de soins de santé, tout en protégeant les Canadiens par le dépistage des maladies infectieuses. De nombreux partenaires gouvernementaux et non gouvernementaux travaillent de concert depuis l'automne 2015 à la mise en œuvre de ce plan.
L'expérience européenne sur l'accueil de migrants sans papiers et en situation «irrégulière » en 2015 a fourni aux autorités canadiennes des renseignements utiles desquels ils ont pu tirer des leçons (1). Cette expérience a notamment démontré que les réfugiés en provenance des régions touchées ne présentaient pas de menace pour les populations intérieures en ce qui a trait aux maladies infectieuses. Ils étaient en revanche vulnérables aux affections qui se propagent facilement dans de mauvaises conditions de vie (2).

Contrairement à l'Europe où la crise des réfugiés syriens a donné lieu à l'entrée de milliers de migrants qui n'avaient pas subi au 
préalable d'examen médical et qui n'avaient pas été inscrits, le Canada a été en mesure d'assurer la mise en place d'un programme de dépistage médical avant l'arrivée des réfugiés. Cette mesure a non seulement contribué à protéger la santé publique, mais a également facilité l'intégration des réfugiés dans le système canadien de soins de santé.

\section{L'accueil des réfugiés : Le dépistage}

De décembre 2015 à février 2016, un total de 26166 réfugiés syriens et membres de leur famille sont arrivés au Canada à bord de 99 vols distincts. De ce nombre, 14992 réfugiés ont été pris en charge par le gouvernement et les 11174 autres étaient soit parrainés par le secteur privé, soit arrivés dans le cadre du Programme mixte des réfugiés désignés par un bureau des visas. Bien que tous les voyageurs aient été soumis à un dépistage à leur arrivée aux aéroports internationaux, la santé des réfugiés à destination du Canada avait aussi été évaluée aux multiples points de départ, avant leur départ.

L'examen médical aux fins de l'immigration (EMI) a été effectué avant le voyage; tous les immigrants potentiels ont été évalués en regard d'une courte liste de maladies transmissibles d'importance pour la santé publique. Si l'une ou l'autre de ces maladies était découverte et que la personne demeurait admissible au statut de réfugié, Immigration, Réfugiés et Citoyenneté Canada a communiqué l'information aux autorités locales de santé publique au Canada afin que la personne fasse l'objet d'un suivi approprié. À ce jour, parmi les réfugiés syriens arrivés dans le cadre des efforts actuels de réinstallation, seulement deux cas ont été signalés aux responsables locaux de la santé publique; il s'agit de deux personnes dont les radiographies pulmonaires réalisées dans le cadre de l'examen médical aux fins de l'immigration ont révélé une possible tuberculose latente (non infectieuse). Les autorités locales de santé publique dirigeront ces personnes vers les services compétents pour que ces personnes obtiennent les soins cliniques requis et fassent l'objet d'une surveillance après leur arrivée. Immigration, Réfugiés et Citoyenneté Canada et l'Agence de la santé publique du Canada collaborent actuellement à l'analyse épidémiologique plus approfondie des données de l'examen médical aux fins de l'immigration.

En plus de l'examen médical aux fins de l'immigration, les réfugiés ont été soumis à un dépistage à leur arrivée au point d'entrée au Canada (aéroports de Toronto et de Montréal), conformément aux responsabilités du gouvernement fédéral en vertu de la Loi sur la mise en quarantaine $(3,4)$. Depuis l'arrivée du premier vol nolisé de réfugiés syriens le 10 décembre 2015, des agents de quarantaine - qui étaient des infirmières et des médecins qualifiés - ont été présents dans les aéroports de Toronto et de Montréal lors de chacun des 99 vols effectués transportant en moyenne 249 passagers. (Pour un compte rendu de première main relatant l'expérience d'un professionnel de la santé, voir l'article écrit par D'Amour dans le présent numéro [5].) Les passagers qui semblaient malades à l'arrivée ou qui étaient susceptibles d'être atteints d'une maladie transmissible ont été dirigés vers des agents de quarantaine pour une évaluation plus détaillée avec le soutien d'un interprète. La plupart de ces personnes étaient des enfants qui arrivaient après un long vol international et qui présentaient de la fièvre, une toux ou des vomissements. À ce jour, les agents de quarantaine ont évalué 274 réfugiés syriens (soit environ 1\%). De ce nombre, dix ont été dirigés vers des hôpitaux locaux pour une évaluation et des soins plus approfondis; aucun ne présentait une maladie transmissible préoccupante pour la santé publique.

Des équipes médicales ont été déployées dans les centres d'accueil des aéroports du Québec et de l'Ontario pour fournir des soins d'urgence; elles ont évalué environ 1212 réfugiés $(4,9 \%)$. Dans la plupart des cas, les équipes médicales d'urgence ont été en mesure d'offrir des soins sur place, et seulement 54 réfugiés ont été transportés à l'hôpital. Les médecins qui accompagnaient les réfugiés à bord des vols nolisés à destination du Canada avaient été informés des voyageurs qui présentaient des problèmes de santé pressants, par exemple une néphropathie, et ils ont facilité l'aiguillage de ces personnes vers des cliniques et des hôpitaux à l'arrivée (A. Boucard, communication personnelle, 25 janvier 2016).

Les données sur l'état de santé des réfugiés syriens à leur arrivée au Canada sont résumées au tableau 1.

Tableau 1 : État de santé des réfugiés syriens à leur arrivée au Canada entre le 4 novembre 2015 et le 29 février 2016

\begin{tabular}{|c|c|c|}
\hline Caractéristique & Nombre & Pourcentage \\
\hline Nombre total de réfugiés & 26166 & $100,00 \%$ \\
\hline Pris en charge par le gouvernement & 14992 & $57,30 \%$ \\
\hline Parrainés par le secteur privé & 11174 & $42,70 \%$ \\
\hline $\begin{array}{l}\text { Maladies à déclaration obligatoire } \\
\text { recensées dans le cadre de l'examen } \\
\text { médical aux fins de l'immigration }\end{array}$ & 2 & $0,01 \%$ \\
\hline $\mathrm{VIH}$ & 0 & $0 \%$ \\
\hline Tuberculose latente & 2 & $0,01 \%$ \\
\hline Syphilis & 0 & $0 \%$ \\
\hline Dépistage soutenu à l'arrivée* & 24640 & $94,17 \%$ \\
\hline $\begin{array}{l}\text { Évalués par l'agent de quarantaine en } \\
\text { raison de signes d'infection à l'arrivée }\end{array}$ & 274 & $1,11 \%$ \\
\hline Dirigés vers un hôpital local & 10 & $0,04 \%$ \\
\hline $\begin{array}{l}\text { Nécessitant des soins d'urgence par le } \\
\text { personnel paramédical à l'arrivée }\end{array}$ & 1212 & $4,92 \%$ \\
\hline Transférés à l'hôpital & 54 & $0,22 \%$ \\
\hline
\end{tabular}

\section{Répondre aux besoins continus des réfugiés syriens en matière de santé}

Les fournisseurs de soins de santé au Canada travaillent de concert afin d'établir une approche concertée qui permettra de répondre aux besoins continus des réfugiés syriens en matière de santé. Les deux provinces qui ont accueilli les premiers réfugiés syriens (Ontario et Québec) ont mis en branle des plans exhaustifs dans le domaine de la santé pour l'accueil et la réinstallation des réfugiés $(6,7)$. Les services locaux de santé publique se sont préparés à répondre aux deux besoins initiaux les plus pressants des réfugiés syriens, soit la mise à jour de la vaccination et les soins dentaires urgents (8-10). La Collaboration 
canadienne pour la santé des immigrants et des réfugiés (CCSIR) a établi, à l'intention des omnipraticiens, une liste de vérification (11) qui énonce des recommandations fondées sur des données probantes sur les moyens d'évaluer les besoins en matière de soins de santé des réfugiés et d'y répondre au cours d'une série de consultations (12).

Il y a eu dans certains cas des lacunes ou des retards dans la prestation des soins de santé, en raison de la difficulté à trouver des logements permanents pour les réfugiés pris en charge par le gouvernement et des défis liés à la prestation des services. Cependant, la décision des premières provinces ayant accueilli des réfugiés de renoncer à la période d'attente de trois mois pour être admissible aux régimes provinciaux d'assurance-maladie, ainsi que le haut degré de collaboration entre les fournisseurs locaux de soins, ont facilité l'intégration des réfugiés dans le système canadien de soins de santé.

On possède peu de données portant expressément sur l'état de santé des demandeurs d'asile et des réfugiés. La $D^{r e}$ Anna Banerji, spécialiste des maladies infectieuses pédiatriques à Toronto, a dispensé des soins à des familles de réfugiés syriens dans les centres d'accueil des immigrants durant la période suivant immédiatement leur arrivée au pays; elle a observé que jusqu'à un tiers des enfants réfugiés étaient atteints de maladies virales courantes comme des infections des voies respiratoires supérieures, des infections de l'oreille et de la gorge et des maladies gastro-intestinales. Une éclosion de grippe a aussi été observée chez 450 réfugiés syriens arrivés à Edmonton, en Alberta, à la fin de janvier 2016 (12). Ces réfugiés étaient arrivés au plus fort de la saison de la grippe saisonnière, avant que tous aient pu recevoir le vaccin antigrippal; environ la moitié des adultes et la plupart des enfants sont tombés malades (13). Ces infections se sont résorbées avec le temps et des soins de soutien.

Conformément à ce qui a été observé en Union européenne, aucun problème de santé mentale grave n'a été signalé à ce jour parmi les réfugiés syriens. Il est probable que des troubles de stress post-traumatique apparaîtront à long terme, à mesure que les réfugiés s'établiront dans leur nouvelle vie et qu'ils seront en mesure de faire face mentalement aux stress de la guerre, du déplacement et de la perte qu'ils ont vécus. Des psychiatres culturels de l'Ontario et du Québec offrent des consultations en ligne dans le cadre d'un effort visant à renforcer la capacité d'offrir des services de santé mentale adaptés aux différences culturelles dans l'ensemble du pays.

On s'attend également à ce que des problèmes de santé chroniques apparaissent au fil du temps. Il est possible que, par crainte, certains réfugiés ne déclarent pas certains problèmes de santé durant le processus d'évaluation (14); on s'attend donc que $5 \%$ des réfugiés par ailleurs "en bonne santé ", qui ont subi un examen médical aux fins de l'immigration, aient des besoins continus pour certaines formes de soins de santé (M. MacKinnon, communication personnelle, 25 janvier 2016). Lorsque le Haut Commissariat des Nations Unies pour les réfugiés (UNHCR) a communiqué avec les autorités canadiennes au sujet des réfugiés, il les a informées que les réfugiés syriens pourraient avoir de "grands besoins en matière de soins médicaux ". Ces besoins pourraient être liés à des problèmes tels que le diabète, des déficiences sur le plan du développement et des traumatismes liés au conflit. La pédiatre affectée au centre d'accueil des immigrants de Toronto est d'accord avec les estimations d'Immigration, Réfugiés et Citoyenneté Canada; elle a observé des problèmes allant de crises d'épilepsie et de troubles du développement à des cas de thalassémie nécessitant des transfusions sanguines et de cancers pédiatriques (A. Banerji, communication personnelle, 25 janvier 2016). Le coprésident de Collaboration canadienne pour la santé des immigrants et des réfugiés a relevé des problèmes de malnutrition, et des médecins ont noté qu'un certain nombre d'enfants avaient des déficiences intellectuelles; il est toutefois trop tôt pour déterminer si l'incidence de ces problèmes est plus élevée que l'incidence moyenne en Amérique du Nord. On est à mettre en place un système de surveillance pédiatrique pour surveiller ces faits (K. Pottie, communication personnelle, 22 février 2016).

\section{Conclusion}

Les mouvements de masse de réfugiés exercent des pressions sur les systèmes de soins de santé, tant sur le plan des interventions immédiates que des efforts de réinstallation à long terme. À mesure que les Syriens s'intégreront à la population canadienne, il faudra pouvoir compter sur un réseau homogène de fournisseurs de soins de santé, appuyés par des partenaires de la communauté pour répondre aux besoins à plus long terme des réfugiés en matière de soins de santé. Il faudra également compter sur l'engagement continu des gouvernements et du milieu universitaire afin de renforcer la capacité du système de santé, en formant des personnes compétentes grâce aux meilleures technologies disponibles et en surveillant les nouveaux problèmes qui surgiront.

L'expérience du Canada au cours des dernières semaines n'a mis en lumière aucune préoccupation majeure en ce qui a trait aux maladies transmissibles. Comme on s'y attendait, les réfugiés syriens arrivés au Canada ont certains besoins non comblés en matière de soins de santé. Le secteur de la santé cherche à satisfaire à ces besoins, mais les fournisseurs de soins de santé et la population générale peuvent avoir l'assurance que les risques pour la santé publique ont été minimes.

\section{Remerciements}

Nous tenons à remercier toutes les personnes qui ont contribué aux efforts visant à accueillir des réfugiés syriens au Canada.

\section{Conflit d'intérêts}

Aucun.

\section{Références}

1. European Centre for Disease Prevention and Control. Communicable disease risks associated with the movement of refugees in Europe during the winter season - 10 November 2015. Stockholm: ECDC; 2015. 
2. Catchpole M, Coulombier D. Refugee crisis demands European Union-wide surveillance! Eurosurveillance. 2015;20(45):2-3.

3. Loi sur la mise en quarantaine. L.C. 2005, ch. 20. http://lawslois.justice.gc.ca/fra/lois/q-1.1/TexteComplet.html.

4. Bhatia N, Sarwal S, Robinson H, Geduld J, Huneault F, Schreiner H, Collins S, Hickey R. Federal public health strategies to minimize the importation of communicable diseases into Canada. Can Comm Dis Rep. 2015;41(S6):3-8. (Disponible en français : http://www.phac-aspc. gc.ca/publicat/ccdr-rmtc/15vol41/dr-rm41s-6/assets/ pdf/15vol41s-6_overview-apercu-fra.pdf).

5. D'Amour R. My experience as a Quarantine Officer welcoming Syrian refugees. Can Comm Dis Rep 2016;42-Suppl 2:S11-2. (Disponible en français : http://www. phac-aspc.gc.ca/publicat/ccdr-rmtc/16vol42/dr-rm42-s2/ar03-fra.ph).

6. Ontario Ministry of Health and Long-Term Care. Ontario Health System Action Plan: Syrian refugees, December 17, 2015. Toronto: MOHLTC; 2015. (Disponible en français : http://www.health.gov.on.ca/fr/pro/programs/emb/ syrianrefugees/docs/health_system_action_plan.PDF).
7. Plan d'action du gouvernement du Québec pour l'accueil et l'intégration des personnes réfugiées sélectionnées à l'étranger 2013-2016. https://www.immigrationquebec.gouv.qc.ca/publications/fr/divers/PLA_ AccueilRefugies2013-2016.pdf.

8. Toronto Public Health. Staff report: Response to Syrian refugee health needs. January 11, 2016. http://www.toronto. $\mathrm{ca} /$ legdocs $/ \mathrm{mmis} / 2016 / \mathrm{hl} / \mathrm{bgrd} /$ backgroundfile-88528. pdf.

9. City of Toronto Public Health. Refugee resettlement program. Toronto: City of Toronto; 2016. http://www1. toronto.ca/wps/portal/contentonly?vgnextoid=93614790451 a1510VgnVCM10000071d60f89RCRD.

10. Refugee 613. Health toolkit for refugees: Ottawa Specific2015/2016. Ottawa ON: Ottawa Newcomer Health Centre and Somerset West Community Health Centre; 2015. http:// www.swchc.on.ca/sites/default/files/Refugee_Health_ Toolkit_2015-2016.pdf.

11. The Canadian Collaboration for Immigrant and Refugee Health. Evidence-based preventative care checklist for new immigrants and refugees. Ottawa: CCIRH; 2016. http://www. ccirhken.ca/ccirh/checklist_website/index.html.

12. Pottie K, Greenaway C, Hassan G, Hui C, Kirmayer L. Caring for a newly arrived Syrian refugee family. CMAJ. 2016;Epub.151422.

13. Syrian refugees hit by first flu season in Canada. Canadian Broadcasting Company. January 22, 2016. http://www.cbc. $\mathrm{ca} /$ news/canada/edmonton/syrian-refugees-hit-by-1st-fluseason-in-canada-1.3415254.

14. O'Donnell CA, Higgins M, Chauhan R, Mullen K. Asylum seekers' expectations of and trust in general practice: A qualitative study. Br J Gen Pract J R Coll Gen Pract. 2008;58(557):e1-11. 